\title{
High mebendazole doses in pulmonary and hepatic hydatid disease
}

\author{
J Messaritakis, P Psychou, P Nicolaidou, T Karpathios, B Syriopoulou, A Fretzayas, \\ F Krikos, N Matsaniotis
}

\begin{abstract}
Thirty nine children with 71 hydatid cysts were given mebendazole orally in a dose of $100-200 \mathrm{mg} / \mathrm{kg} /$ day for 12 weeks and were followed up for a mean (SD) of 63 (24) months. Twenty children (three of them after a second course) were cured and another two avoided at least one operation. No serious side effects of the drug were observed.
\end{abstract}

Experience during the last 10 years with several benzimidazole derivatives, mainly mebendazole and albendazole, suggests that chemotherapy may, in certain circumstances, replace surgery in the treatment of hydatidosis in children. ${ }^{12}$ In this study the effectiveness of mebendazole has been evaluated prospectively.

4

Patients, methods, and results

Thirty nine children ( 17 boys and 22 girls) aged 2-14 years (mean age 7.5 years) with cystic hydatidosis received mebendazole orally for 12 weeks $(100-200 \mathrm{mg} / \mathrm{kg} /$ day with a maximum daily dose of $6 \mathrm{~g}$ ) in divided doses with meals. Nine patients who failed to respond received a second course of treatment after an interval of three to six months. The 39 patients had 71 cysts: 33 in the lungs (seven of which had ruptured), 37 in the liver, and one in the spleen. The patients were followed up during the treatment to observe any haematological, hepatic, renal, visual, and hearing problems because of toxicity.

The results of the treatment were evaluated according to the organ involved by radiography, computed tomography, or ultrasonography, and were classified into cure (disappearance of the cysts), improvement (decreased size of the cyst and/or calcification or various alterations in density and/or negative viability test), and failure.

The results are summarised in the table. The mean (SD) follow up period for the successfully treated cysts was 63 (24) months. The successfully treated lung cysts were smaller (mean (SD) diameter $5.24(1.92) \mathrm{cm})$ than those which persisted $(7.94(4.38) \mathrm{cm}, \mathrm{p}<0.05)$. Similar

Results of the mebendazole treatment $(n=70)^{*}$

\begin{tabular}{lcl}
\hline & Lungs & Liver \\
\hline No of cysts & 33 & 37 \\
Cure (\%) & $22(67)$ & $10(27)$ \\
Improvement (\%) & $2(6)$ & $11(30)$ \\
Failure (\%) & $9(27)$ & $16(43)$ \\
\hline
\end{tabular}

*There was one cyst found in the spleen, which did not respond to treatment. differences were also found in the hepatic cysts $(3.93(1.87)$ and $7.78(3.08) \mathrm{cm}$, respectively, $\mathrm{p}<0.001)$.

Of the 39 patients 20 (three of them after a second course) were cured and two with multiple cysts avoided at least one operation. Nine of the 20 cured patients had multiple cysts in one or more organs.

The mean (SD) plasma mebendazole concentration two hours after the morning dose was 116 (47) $\mathrm{ng} / \mathrm{ml}$. Serious side effects from mebendazole were not observed. Seven children had a low grade fever, two children had gastric discomfort, and two others had a transient moderate rise of transaminase activity.

\section{Discussion}

Mebendazole, a synthetic benzimidazole derivative, is effective against the larval stages of Echinococcus granulosus and $E$ multilocularis. It kills the parasite by limiting its glucose uptake through the disruption of the microtubles in the germinal membrane cells of hydatid cysts. ${ }^{3}$

During the last decade, mebendazole has been on clinical trial, especially in adults, as a non-surgical treatment of human hydatid disease. ${ }^{14}$ As far as we know this is the largest series in children with the longest follow up period.

The effects of mebendazole treatment in the literature are not constant. Several factors including drug dose, duration of treatment, age of the patients, size and location of the cysts, and duration of follow up may be responsible for this controversy. ${ }^{14}$ The results of this study are considered as favourable, because about two thirds of the pulmonary and one third of the hepatic cysts disappeared without any relapse.

The high effectiveness of mebendazole in this study could be attributed to the higher doses of the drug used and/or the younger age of the patients. In our first published paper we successfully used a dose of $100 \mathrm{mg} / \mathrm{kg} /$ day in only two patients. 5 The absence of serious side effects of mebendazole enabled us to use a dose of $100-200 \mathrm{mg} / \mathrm{kg} /$ day for the 39 cases of the present series. With the above dose of mebendazole we achieved therapeutic plasma concentrations $(100 \mathrm{ng} / \mathrm{ml})$, although they were measured two hours after the morning dose instead of four. ${ }^{6}$ On the other hand, it is known that the efficacy of mebendazole is better in young age. ${ }^{4}$ The assumption is that younger patients tend to have rather smaller and younger cysts. Furthermore in our study, as in others, ${ }^{4}$ the efficacy of the drug was correlated with the size of the cysts. Cysts with a diameter of $4-5 \mathrm{~cm}$ responded better to treatment. The common 
denominator of the age of the patient and the size of the cyst is probably the thickness of cystic adventitia, which is the barrier mebendazole should penetrate in order to reach the germinal layer. The cyst's adventitia is a local reaction of host tissue and pulmonary adventitias are thinner than those of liver cyts. ${ }^{4}$ This could explain the better results in the treatment of pulmonary cysts obtained by us and others. ${ }^{1}$

We have therefore been able to show in this study that high doses of mebendazole have been effective against hydatidosis of children especially in cysts of medium and small size, without serious side effects, and can be used as an alternative treatment.
1 Davis A, Pawlowski ZS, Dixon H. Multicentre clinical trials of benzimidazole carbamates in human echinococcosis. Bull WHO 1986;64:383-8.

2 Todorov T, Vutova K, Petkov D, Mechkov G, Kolev K. Albendazole treatment of human cystic echinococcosis. Albendazole treatment of human cystic
Trans $R$ Soc Trop Med Hyg 1988;82:453-9.

3 Kammerer WS, Judge DM. Chemotherapy of hydatid disease (Echinococcus granulosus) in mice with mebendazole and bithionol. Am f Trop Med Hyg 1976;25:714-7.

4 Gil Grande LA, Boixeda D, Garcia-Hoz F, et al. Treatment of liver hydatid disease with mebendazole: a prospective study of thirteen cases. Am $\mathcal{J}$ Gastroenterol 1983;78:584-8.

5 Karpathios T, Syriopoulou V, Nicolaidou P, Messaritakis J. Mebendazole in the treatment of hydatid cysts. Arch Dis Child 1984;59:894-6.

6 Woodtli W, Bircher J, Witassek F, Eckert J, Wuthrich B Amman RW. Effect of plasma mebendazole concentrations in the treatment of human echinococcosis. Am $\mathcal{J}$ Trop Med Hyg 1985;34:754-60.

\title{
Familial growth hormone releasing factor deficiency in pseudopseudohypoparathyroidism
}

\author{
H F Stirling, D G D Barr, C J H Kelnar
}

Department of Child Life and Health,

University of Edinburgh,

15-17 Hatton Place,

Edinburgh EH9 1UW

H F Stirling

D G D Barr

C J H Kelnar

\section{Correspondence to:}

\section{Dr Stirling.}

Accepted 12 November 1990

(Arch Dis Child 1991;66:533-5).
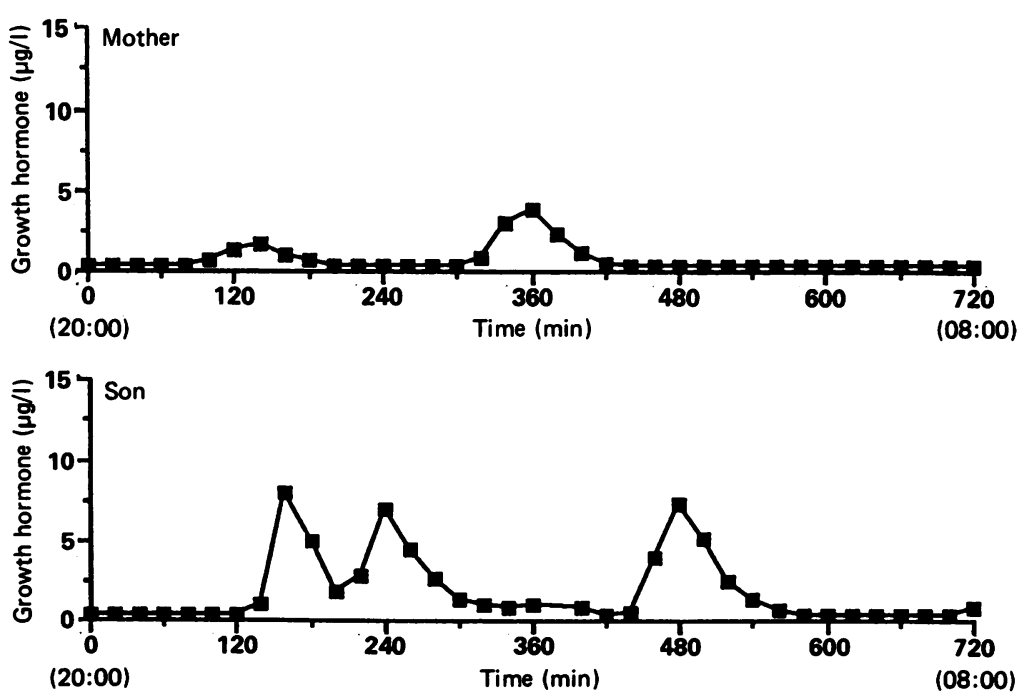

Figure 1 Overnight growth hormone secretion in mother and son. (Growth hormone comversion factor, $1 \mu \mathrm{g} / \mathrm{l}=2 \mathrm{mU} / \mathrm{l}$.)

\begin{abstract}
A mother with pseudopseudohypoparathyroidism and her short son showed poor spontaneous growth hormone secretion, and provocation tests suggested a deficiency of growth hormone releasing factor. This is the first report of growth hormone releasing factor deficiency in pseudopseudohypoparathyroidism. The boy has responded well to growth hormone treatment over a period of three years.
\end{abstract}

\section{Case reports}

A 4.5 year old healthy boy presented with short stature (height standard deviation score (SDS)

08:00)
$-3.93)$. His mother was short $(145 \cdot 2 \mathrm{~cm})$ and had clinical features of pseudopseudohypoparathyroidism (short fourth and fifth metacarpals and metatarsals, round facies). She was euthyroid but in the past had been treated with carbimazole and radioactive iodine for hyperthyroidism. The child had normal thyroid function. Both the boy and his mother were normocalcaemic with normal plasma cyclic AMP responses to parathyroid hormone stimulation.

Both mother and son had poor spontaneous overnight growth hormone secretion (fig 1). One would have expected three to four pulses of growth hormone during the night with pulse amplitude $>10 \mu \mathrm{g} / 1$ in the child. The mother failed to produce any significant growth hormone pulses. The boy's response to insulin induced hypoglycaemia was poor with a peak growth hormone concentration of $4 \cdot 1 \mu \mathrm{g} / \mathrm{l}$. The cortisol response was satisfactory. However when given growth hormone releasing factor both mother and child showed a very pronounced response: the child with a peak growth hormone concentration of $>95 \mu \mathrm{g} / \mathrm{l}$ and the mother with a concentration of $73 \mu \mathrm{g} / \mathrm{l}$.

Since the age of 5.7 years the boy has been treated with growth hormone ( $24 \mathrm{IU} / \mathrm{m}^{2} /$ week). His height SDS at the start of growth hormone treatment was $-3 \cdot 70$ with a height velocity of $4.9 \mathrm{~cm} /$ year (height velocity SDS -1.47 ). Height velocity over the first year of treatment increased to $9.1 \mathrm{~cm} /$ year (height velocity SDS $+3 \cdot 28$ ). Good growth has been maintained on growth hormone for three years and he now has a height SDS -1.95 (fig 2). Bone age has not advanced abnormally and his final height prognosis has improved. 